\title{
L'ANALYSE POLLINIQUE DES MIELS DE LA RÉPUBLIQUE POPULAIRE ROUMAINE
}

\author{
PAR
}

\section{Dr. Cornelia PELIMON}

Bucarest

Les ressources mellifères de la République populaire de Roumanie seraient suffisantes pour nourrir dix fois l'effectif des 650 ooo ruches qui y existent actuellement. De très grandes possibilités de récolte nous sont offertes par la transhumance des ruches vers les peuplements mellifères naturels et stables ainsi que vers les différentes cultures dont les implantations sont susceptibles de varier en fonction des nécessités de la nouvelle agronomie. Dans ces cultures, on vise d'ailleurs aussi bien à la production du miel qu'à la fécondation des fleurs par les abeilles.

Nos ressources mellifères essentielles sont constituées par de vastes peuplements de Robinier faux-acacia, de Tilleul, de Ronce, d'Epilobe, de Menthe, ainsi que par des prairies et des étendues limitées de Tamaris le long de plusieurs rivières. Nous possédons aussi quelques vieilles plantations de Châtaigniers. Dans toute la région des collines, en plus des arbres fruitiers, il y a des cultures locales : Sarrasin, Tournesol, Cucurbitacées, des plantes médicinales et à parfum ainsi que du coton et de la Phacélie. I a carte ci-jointe (fig. I) indique l'emplacement des principales ressources mellifères.

Robinia pseudacacia constitue la principale ressource mellifère printanière des régions de la plaine. Il occupe en tout 80 ooo ha sous la forme de forêts et de rideaux d'arbres. Il faut aussi mentionner les plantations qui se trouvent dans la steppe (Baragan) aux environs de Braila, Hanul Conachi (région administrative de Galati), dans le sud de la région de Craiova, à Alios (district d'Arad) et Valea lui Mihai dans la région d'Oradea.

Tilia. Il convient pour les Tilleuls de distinguer plusieurs massifs forestiers importants :

a) Au nord de la Dobroudgea, entre le Danube et la Mer Noire 





entre 205 et $456 \mathrm{~m}$ d'altitude, le Tilleul représente $50 \mathrm{p}$. Ioo du peuplement forestier mélangé de 50 ooo ha, fait considéré comme unique en Europe.

b) Dans les forêts mélangées de Iasi, Husi, Vaslui, Negoesti, Tg. Frumos, Bîrlad, Murgeni, Roman, Hîrlau, le Tilleul occupe une surface de 22000 ha.

c) Dans le sud-ouest de la région de Timisoara, le Tilleul occupe 9 ooo ha dans des forêts à plusieurs essences (Resita, Oravita, Moldova Noua, Orsova, Lipova, Ceahova, Caransebes).

d) Dans la région de Bucarest, le Tilleul occupe une étendue de 7 ooo ha dans les forêts à essences mélangées de Snagov, Domnesti, Titu, Videle, Branesti, Vidra, Comana.

Dans le reste du pays, Tilia se trouve sur de petites surfaces.

Castanea sativa. Les plantations de Châtaigniers modifient l'aspect habituel de la végétation près de Tismana (district de Baia de Arama) sur une étendue de 30 ha ainsi que près de Seini (région administrative de Baia Mare) et sur divers points tels que Caransebes, Cuvin (district de Arad), Hunedoara, Prejmer (région administrative de Stalin).

Dans les régions montagneuses, Rubus et Epilobium embellissent les pentes ensoleillées et assurent quelquefois aux abeilles une bonne récolte. Mille fleurs existent dans les prairies de montagne, constituant la première et la principale récolte pour les ruches de ces contrées.

$\mathrm{Au}$ sud, dans la "Balta ", c'est-à-dire dans les régions inondables du Danube, les Saules foumissent en abondance au printemps du pollen et du nectar. La Menthe, Trifolium minus, etc... qui constituent la flore spécifique, fleurissent ensuite de juillet à septembre.

En ce qui concerne l'analyse pollinique des miels, un seul travail a été publié dans notre pays, celui de A. FARCAs, "Recherches sur le pollen des arbres fruitiers dans le miel " (C1uj I939). Entre I940 et I95I, après des travaux préparatoires qui ont abouti à une collection de préparations permanentes de 400 espèces de pollen, on a examiné à 1'Institut de recherches zootechniques de Bucarest 257 échantillons de miel provenant de toutes les régions du pays. La technique employée a été celle de E. ZANDER. On s'est procuré les échantillons directement dans les ruchers et, en plus petit nombre, dans le commerce. Il s'agissait, soit de miels multifloraux récoltés sur l'ensemble de la saison apicole, soit de miels unifloraux.

La figure 2 représente sous forme d'un graphique les principaux résultats de nos analyses polliniques. Pour chaque pollen nous indiquons, d'une part le maximum du pourcentage relevé dans les échantillons unifloraux et d'autre part la fréquence avec laquelle se présentent les différents pollens dans les 257 échantillons analysés. 
Dans la première colonne on trouve Trifolium. Il s'agit de pollens de Trèfles mais à l'exception de $T$. pratense et $T$. incarnatum, lesquels n'ont été identifiés que très rarement et seulement comme pollens isolés. Les pollens de Trèfle sont les plus fréquents dans les miels roumains puisqu'on les a trouvés dans 93 p. Ioo des échantillons. Il s'agit de Trèfles

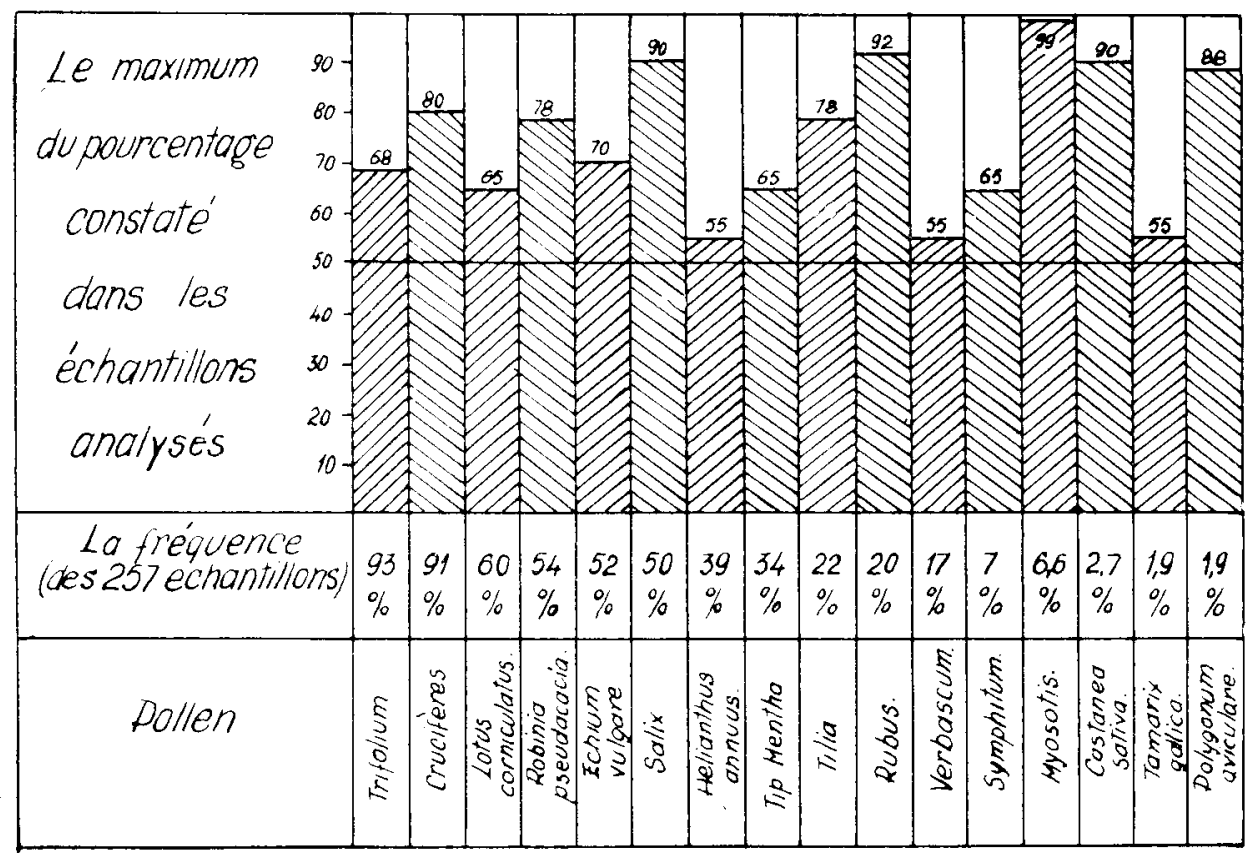

FiG. 2.

vivant sur des terrains incultes ou peu cultivés. Le pourcentage maximum dans les miels de Trèfles unifloraux atteint 68 p. Ioo.

Dans la deuxième colonne suivent les Crucifères, comprenant les genres Brassica, Sinapis, ainsi que les espèces Raphanus raphanistrum, Cardamine pratensis, avec la fréquence de $9 \mathrm{I}$ p. Ioo et le maximum de pollen de 80 p. Ioo. Dans la majorité des cas il s'agit de plantes spontanées et, très rarement, de plantes cultivées.

Robinia atteint une fréquence de $54 \mathrm{p}$. roo et Tilia seulement de 22 p. Ioo. Tous deux ont un maximum de pollen de $7^{8}$ p. Ioo. Ces chiffres montrent qu'il s'agit de plantes présentes sur des surfaces limitées. Tilia est très loin derrière Trifolium.

Lotus corniculatus existe partout. Echium vulgare démontre aussi sa valeur de plante mellifère sauvage. Le pollen de Salix a une fréquence de $50 \mathrm{p}$. roo des échantillons, ce qui rappelle l'existence de cet arbuste dans toutes les régions et surtout le long des rivières et du Danube. 
Helianthus annuus, Mentha, Rubus, Symphytum, Tamarix, ont une fréquence plus limitée. Le maximum de pollen est au-dessus de 46 p. roo mais très près de cette limite, c'est-à-dire pratiquement entre pollen dominant et pollen secondaire. Tamarix ne joue qu'un rôle secondaire quant à son influence sur les caractéristiques du miel. L'échantillon de miel unifloral de Rubus constitue une exception en ce qui concerne le pollen, avec le maximum très élevé de $92 \mathrm{p}$. Ioo. En dehors de Rubus les autres plantes (Helianthus, Mentha, Symphytum, Tamarix) n'ont présenté qu'un seul échantillon où le pollen soit dominant et peu au-dessus d'ailleurs de la valeur limite. Dans les autres échantillons ils étaient dans la classe des pollens secondaires.

Les échantillons de miels unifloraux se distinguent par quelques caractères particuliers. Ainsi le miel de Saule, qui représente une ressource exceptionnelle ( $3 \mathrm{~kg}$ par jour), a été récolté à part $1 \mathrm{l} 4$ avril. Ce miel était clair, nuancé très légèrement de vert et présentait un fin arôme de l'écorce de l'arbuste qui le produit. Dans le culot de centrifugation on a découvert aussi du pollen de Rubus et d'Epilobe provenant du nid à couvain; c'était un reste de l'été précédent qui n'avait pas été consommé pendant l'hiver. Habituellement le miel de Saule se trouve en petite quantité et on ne le récolte pas; on le laisse comme nourriture pour le développement du couvain.

Verbascum a été représenté dans I 7 p. Ioo des échantillons avec comme maximum 55 p. roo dans un miel de Baragan. Ce résultat nous permet de supposer que dans certaines conditions atmosphériques Verbascum peut présenter une abondante sécrétion nectarifère et donner une récolte.

Le pollen de Myosotis ne s'est retrouvé que dans 6,6 p. Ioo des échantillons mais avec un maximum absolu de $99 \mathrm{p}$. Ioo. Ce pourcentage, qui correspond à une importante quantité de nectar, justifie 1'appellation de mial de Myosotis (Maurizio I940).

Les miels d'Epilobe ont montré seulement une petite quantité de pollen : 9, I5, 29 p. Ioo.

Parmi les pollens secondaires on a identifié le pollen assez caractéristique de Loranthus europaeus (plante parasite des Hêtres, Chênes et Bouleaux) avec un maximum de 35 p. Ioo; ce pollen a été signalé par Maurizio (I960). Nous l'avons trouvé dans les miels de I3 localités différentes de la région des collines et une fois en plaine à Calarasi (voir fig. 3).

Les pollens de Fagopyrum esculentum, Corylus avellana, Almus, Anthyllis vulneraria, Frangula, Gossypium, Cucumis, Cucurbita pepo, n'ont pas dépassé la limite supérieure du pollen isolé, soit $15 \mathrm{p}$. Ioo.

Parmi les plantes pollénifères, il faut mentionner dans le groupe "secondaire": Cannabis (3I p. I00), Papaver (20 p. I00), Chenopodium (I8 p. I0o), Rumex (I7 p. I00). 
Dans le culot de centrifugation des miels nous avons constaté aussi la présence de grains d'amidon dans $7 \mathrm{I}, 6$ p. Ioo des échantillons; en général ils sont présents en même temps que des spores de champignons microscopiques. Dans 0,5 p. Ioo des échantillons les grains d'amidon

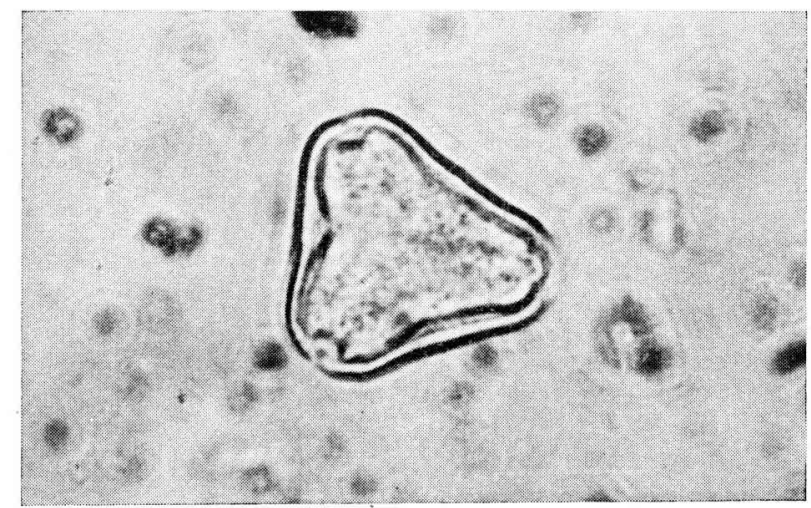

FIG. 3. - Pollen de Loranthus europaeus.

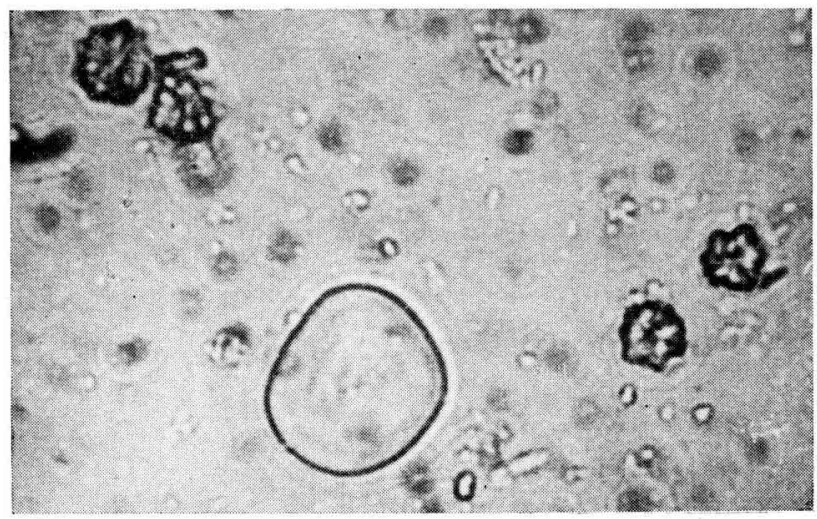

Tici. 4. - Granule d’amidon et cristaux en rosette.

étaient aussi nombreux que les grains de pollen et dans I6 p. Ioo ils étaient plus nombreux.

Nous avons observé dans les miels de forêt des cristaux de différentes formes voisinant avec le pollen de Tilia et accompagnés par les pollens de Myosotis, Antriscus et Trifolium. Des cristaux en forme de rosette (fig. 4) ont été trouvés dans les miels du massif de Tulcea (Dobroudgea). Une substance minérale verte a coloré le sédiment et les cendres d'un miel de prairie. La couleur verte a persisté 2 à 3 jours et a viré ensuite au brun.

Nous avons identifié des Algues vertes dans les miellats, en même 
temps que les spores de champignons microscopiques. Dans un échantillon de miellat presque pur de Sorghum, les spores se trouvaient en nombre égal avec les grains de pollen.

Une masse de fins granules a pu être observée dans les échantillons des miels de la "Balta " mais aussi dans un échantillon de miel multifloral d'Euphorbia, Robinia et Crucifères en provenance de Baragan.

\section{Conclusions}

Entre I940 et I95I, l'examen microscopique du culot de centrifugation de 257 échantillons de miel a mis en évidence la flore mellifère principale de notre pays dont la plus grande partie est constituée par des plantes spontanées; les plantes cultivées sont moins importantes.

L'importance des pollens de Trifolium (avec 93 p. Ioo), des Crucifères, de Lotus corniculatus, Echium vulgare, a démontré le rôle essentiel joué par ces plantes comme source de miel. Helianthus annuus et Brassica (peu important) ont été les seules plantes de culture auxquelles on a pu attribuer des miels unifloraux.

Les échantillons des miels unifloraux de Robinia, Tilia, Epilobium, Rubus, Myosotis, Mentha correspondent aux peuplements indiqués sur la carte.

A la suite de l'examen organoleptique, on a constaté que les miels de Robinia et de Tilia sont plus importants que ne laissait croire la fréquence du pollen dominant à la limite de $46 \mathrm{p}$. Ioo. Ceci confirme les constatations de ZaNDER (I935 à I949), MAUrizio (I9.36 à I960), Príssch (I957).

Les miels de Myosotis, Castanea, Salix, Polygonum aviculare, doivent être considérés comme relativement exceptionnels.

Bien que les peuplements de Menthe au sud du pays s'étendent sur une grande superficie, dans un seul cas le pollen de cette plante a pu être trouvé dans le groupe dominant.

Les cultures d'Helianthus, Gossypium, Polygonum esculentum, Phacelia se sont peu remarquées dans le culot de centrifugation des échantillons examinés. I,e pollen des Arbres fruitiers et des Cucurbitacées constitue une rareté.

On remarque une ressemblance entre le miel roumain et celui de la R. P. de Hongrie en ce qui concerne les miels de Robinia pseudacacia (le plus souvent de couleur claire), de Stachys (dans le nord-ouest du pays) et, en général, par la présence du pollen de Loranthus europaeus.

Les miels roumains de Tilleul ressemblent aussi aux miels de 1'U. R. S. S. par le pourcentage élevé du pollen de Tilia dans le culot de centrifugation. Il en est de même pour le miel d'Epilobium qui est récolté en abondance dans le pays voisin. 
Il y a des différences en comparaison avec les miels de la Suisse, de l'Allemagne et de la Pologne. Nous n'avons constaté la présence d'aucun grain de pollen de Callune ni de Rhododendron. On n'a jamais trouvé de pollen de Taraxacum comme dominant; donc nous n'avons pas de miel de Taraxacum.

Le miel de Polygonum esculentum nous différencie de 1'U. R. S. S. Alors que chez nous il y a peu de cultures de sarrasin au nord-est du pays, en U. R. S. S. cette plante est très cultivée pour la consommation et pour la nourriture des animaux.

L'examen microscopique a confirmé aussi que le miel roumain de couleur claire provient, dans la majorité des cas du nectar des fleurs et non du miellat.

Nous considérons qu'à l'heure actuelle le spectre pollinique des miels de notre pays a pu se modifier légèrement par rapport à celui de I940I95I, ceci en raison de l'extension des cultures de Tournesol et de Coton. Mais la flore spontanée, surtout celle des peuplements mellifères naturels, garde sa position et sa valeur prédominante.

\section{RÉFÉRENCES BIBLIOGRAPHIQUES}

Armbruster et Oenicke. - Die Pollenformen als Mittel zur Honigherkunftbestimmung. Karl Wachholz Verlag. Neumünster in Holstein, I929.

FотA (I.). - Flora de castan comestibil, o importanta sursa nectarifera. Revista Apicultura (2), 1957.

Gluhov (M.). - Plantele melifere cele mai importante si mijloace de inmultirea lor in limba rusa, Moscova, I950.

Grieber (C.). - Zur Pollenanalyse des Honigs. Z. f. Unsers. Lebensmit., 59, 63-79, I97, 44I; 61, 24I, I930-3I.

GRIEBEL (C.). - Mikroskopische Untersuchung des Honigs. Handbuch der Lebensmittelchemie, p. 362-379, I938.

Madrizio (A.). - Gibt es Lindenhonig in der Schweiz. S. B. Z., 59 (3), I48, I 936 .

Maurizio (A.). - Weidenhonig. S. B. Z., 59 (Io) ; 547, I936.

Maurizio (A.). - Pollenanalytische Beobachtungen I-5, S. B. Z., 61, 7 I2, I 938 .

Maurizio (A.). - Untersuchungen zur quantitativen Pollenanalyse des Honigs. Mitt. aus Geb. Lebensm. Hyg., 30, 27-72, I939.

Maurizio (A.). - Vergissmeinnichthonig, S. B. Z., 63, I47, 1940.

Maurizio (A.). - Honig der Edelkastanie. S. B. Z., 64, 409, I94I.

Maurizio (A.). - Pollenanalytische Beobachtungen 6-9. Ber. S. Bot. Ges., 51, 77-95, I94I.

Maurizio (A.). - - Pollenanalytische Beobachtungen Io-12. S. B. Z., 65, 524, I942.

Maurizio (A.). - Pollenanalytische Untersuchungen an Honig und Pollenhöschen. Beiheft S. B. Z., 2 (I8), 320 et 2 (20), 486, I949 et I953.

Maurizio (A.). - Pollenanalytische Beobachtungen 13-I6. Ber. Schw. Bot. Ges., 66, II8-I33, I956.

MAURizio (A.). - Das mikroskopische Bild jugoslawischer Importhonige. Z. f. Bienenforschung, $\mathbf{5}$ (I), 8, I960. 
MELICA (C.). - Introducerea si raspîndirea salcîmului în tara noastra. Revista Apicultura (5), 1957.

Popovici (V.). - Raspîndirea si importanta melifera a teniului din padurile tarii noastre. Revista apicultura (I2), I957.

PRITSCH (G.). - Zum Problem der mikroskopischen Pollenanalyse des Bienenhonigs. Wissens. Zeitsch. der Humbolt Univ, zu Berlin. Math. Naturwis. Reihe. Jg., VI, (2), I97-204, 1956-57.

Stamatelache (D.). - Padurile de tei o bogatie melifera a patriei noastre. Revista Apicultura (7), I957.

Tarnavschi (I.) et Mitroiu (E.). - Studiul morfologic al polenului. Bucuresti, I959.

ZaNDER (E.). - Beiträge zur Herkunftsbestimmung bei Honig. Bd. I. Verlag der Reichsfachgruppe Imker. Berlin I935. - Bd. 2. Liedloff, Loth u. Michaelis, Verlag. Leipzig I937. - Bd. 3. Liedloff, Loth u. Michaelis, Verlag. Leipzig I94I. - Bd. 4. Franz Ehrenwirth Verlag. München I949. 